\title{
Single-center study of Lynch syndrome screening in colorectal polyps
}

\author{
FangChao Zhu', Da Pan ${ }^{1}$, Hui Zhang ${ }^{1}$, Qiong Ye ${ }^{2}$, PeiSong $\mathrm{Xu}^{3}$ and Jie Pan ${ }^{1 *}$ (D)
}

\begin{abstract}
Background: Lynch syndrome is the most common hereditary colorectal cancer syndrome, and adenoma is one of the important premalignant lesions to colorectal cancer in Lynch syndrome. The first objective of this study was to calculate the detection rate of Lynch syndrome in colorectal polyps by using mismatch repair immunohistochemistry as the initial screening strategy. The second objective of this study was to optimize screening strategies for adenoma associated with Lynch syndrome by integrating polyp and/or patient characteristics such as polyp size, location, dysplasia, age of onset and/or family history of cancer.

Methods: From June 2014 to May 2016, immunohistochemistry was performed for mismatch repair proteins (MLH1, MSH2, MSH6, and PMS2) using endoscopically resected specimens obtained from newly diagnosed colorectal adenomas. Gene analysis was performed in patients with missing expression of mismatched repair protein.

Results: Five hundred and ten patients underwent colorectal polyp resection, with a total of 718 polyps. Five hundred and eight resected adenomas underwent mismatch repair protein immunohistochemical testing. Loss of mismatch repair protein expression was observed in six adenomas, accounting for $1.18 \%$ of all adenomas. Five patients then underwent genetic tests to identify two pathogenic mutations from different individuals, while another patient was suspected to have a pathogenic mutation. Three patients were younger than 50 years old. Two patients had advanced histology (high-grade dysplasia and malignant components) and one patient had a family history of cancer.

Conclusion: Immunohistochemical detection of colorectal polyp mismatch repair protein as Lynch syndrome screening efficiency is low. Effective screening strategies may be improved by optimizing patient/polyp selection, such as focusing on young adenoma patients with a family history of cancer, or patients who present with high-risk features (large size, villous, high-grade dysplasia and malignant components).
\end{abstract}

Keywords: Lynch syndrome, Mismatch repair deficiency, Colorectal polyps, Immunohistochemistry, Genetic testing, Screening

\section{Introduction}

Lynch syndrome (LS) is the most common disease that causes hereditary colorectal cancer syndrome, accounting for approximately $2-5 \%$ of all colorectal cancers [1], caused by autosomal dominant inheritance of a germline mutation in DNA mismatch repair (MMR) genes (MLH1, $M S H 2, M S H 6$, and PMS2) or EPCAM gene deletion. Immunohistochemistry (IHC) examining loss of expression (LOE) of one or more MMR proteins can be performed on adenomas as a screening test for LS, and subsequent

\footnotetext{
* Correspondence: 26996360@qq.com

'Department of gastroenterology, WenZhou Central Hospital, WenZhou,

ZheJiang Province, China

Full list of author information is available at the end of the article
}

genetic testing based on MMR IHC can ultimately confirm an LS diagnosis.

Colorectal adenoma is one of the most important precancerous lesions of colorectal cancer in LS. Compared with sporadic colorectal cancer, LS related adenoma can easily develop into invasive malignant tumor and accelerate canceration [2]. Adenomas in MMR mutation carriers have rates of MMR IHC deficiencies from 50 to $80 \%$ [3-5]. There seems to be a possibility of MMR IHC as a screening test for LS for premalignant polyps. However, some studies suggest the rate of LOE in MMR proteins or microsatellite instability (MSI) in sporadic adenomatous polyps is low $(<2 \%)$ [6-8]. Loukola et al. reported the frequency of MSI in adenomas was $1.6 \%$ 
(6/378); and in an unselected Finnish population, five out of six patients (83\%) exhibited a germline MMR gene mutation [6]. In a recent study of 57 patients, the incidence of abnormal MMR IHC was 5.3\%, but most of these patients had a family history of cancer or polyps [9]. The incidence of MMR IHC abnormalities in young patients was also low $[7,10]$. The study on the LOE of MMR protein in Chinese polyps is not clear, so we have carried out MMR IHC, and genetic analysis of patients with LOE of MMR protein.

\section{Materials and methods}

\section{Patient selection}

Between June 2014 and May 2016, Five hundred and ten newly diagnosed colorectal polyps underwent endoscopic polypectomy. The clinicopathological data and family history of cancer were obtained from electronic medical records.

\section{IHC for mismatch repair proteins}

If a patient presented more than one adenoma, we chose the more advanced adenoma for MMR IHC testing. Five hundred and eight adenomas from 508 patients were analyzed by MMR IHC; however, one patient had an inflammatory polyp and one a hyperplastic polyp. IHC was performed on four MMR proteins, MLH1, MSH2, MSH6, and PMS2, using a Staining Automat (Benchmark), according to the manufacturer's protocol. We obtained $4-\mu \mathrm{m}$-thick formalin fixed paraffin sections. Primary antibodies used to detect MMR proteins are included antihMLH1 antibody (clone ES05; ZSGB-Bio Ltd., Beijing, China; 1:50), anti-hMSH2 antibody (clone RED2; ZSGBBio Ltd., Beijing, China; 1:100), anti-hMSH6 antibody (clone UMAB258; ZSGB-Bio Ltd., Beijing, China;1:100), and anti-hPMS2 antibody (clone MOR4G; ZSGB-Bio Ltd., Beijing, China; 1: 40). Nuclear staining of non-tumor cells such as normal colonic epithelial cells, lymphoma cells, or stromal cells served as an internal positive control. We judged that there was no nuclear staining at all in tumor cells and suggested that MMR was deficient. Staining results were assessed by consensus by two independent pathologists (Qiong Ye and Yi Jiang).

\section{DNA extraction and sequencing}

Patients with a defect mismatch repair (dMMR) polyp were referred to as genetic counseling, and informed consent was signed for gene detection. DNA was extracted from leukocytes using the QIAamp DNA Mini kit (Qiagen GmbH, Hilden, German). Germline testing was targeted towards the MMR IHC pattern. Specific primer synthesis and Sanger sequencing were performed by HangZhou Zhiyuan Medical Laboratory Co., Ltd. Amplified DNA fragments included coding exons, flanking intron regions, and promoter regions. Four patients underwent Sanger sequencing by using an ABI 3730XL fully automated DNA sequencer (Thermo Fisher Scientific Inc.). Only one patient underwent next-generation sequencing (NGS) by Beijing Deyidongfang Translational Medicine Research Center Co., Ltd. InSiGHT, and at the same time, ClinVar databases were scanned to determine the significance of mutation.

\section{Statistical analysis}

Data were expressed as medians and ranges. SPSS19.0 software was used for statistical analysis.

\section{Results}

\section{Clinicopathologic characteristics}

Five hundred and ten patients underwent colorectal polyp resection between June 2014 and May 2016. There were 375 men and 135women. The median age of the patients was 60 years (range: 28-89). Seven hundred eighteen polyps were resected, including 343 patients with one polyp, and 167 patients with more than one polyp. The pathological features of the patients included in this study are summarized in Table 1 . The histological types were as follows: tubular adenoma (578 cases), villous adenoma (including tubulovillous) (107 cases), serrated adenoma (6 cases), inflammatory polyp (15 cases) and hyperplastic polyp (12 cases). Five adenomas presented malignant components. The site of the polyps was distributed as follows: left colon (proximal to splenic flexure), 496 cases; right colon (splenic flexure and distal to the splenic flexure), 222 cases.

\section{IHC for MMR proteins}

Clinicopathologic characteristics of six patients with LOE of MMR proteins are summarized in Table 2. LOE of MMR proteins was observed in 6 adenomas accounting for $1.18 \%$ of the patient population.

Table 1 Pathological characteristics of colorectal polyps

\begin{tabular}{ll}
\hline Category & Number \\
\hline Histologic classification & 691 \\
Adenoma & 578 \\
Tubular adenoma & 6 \\
Serrated adenoma & 107 \\
Villous adenoma (including tubulovillous) & 15 \\
Inflammatory polyp & 12 \\
Hyperplastic polyp & 44 \\
High-grade dysplasia & 5 \\
Malignant polyp & \\
Location & 496 \\
Left colon (proximal to splenic flexure) & 222 \\
Right colon (splenic flexure and distal to the splenic flexure)
\end{tabular}




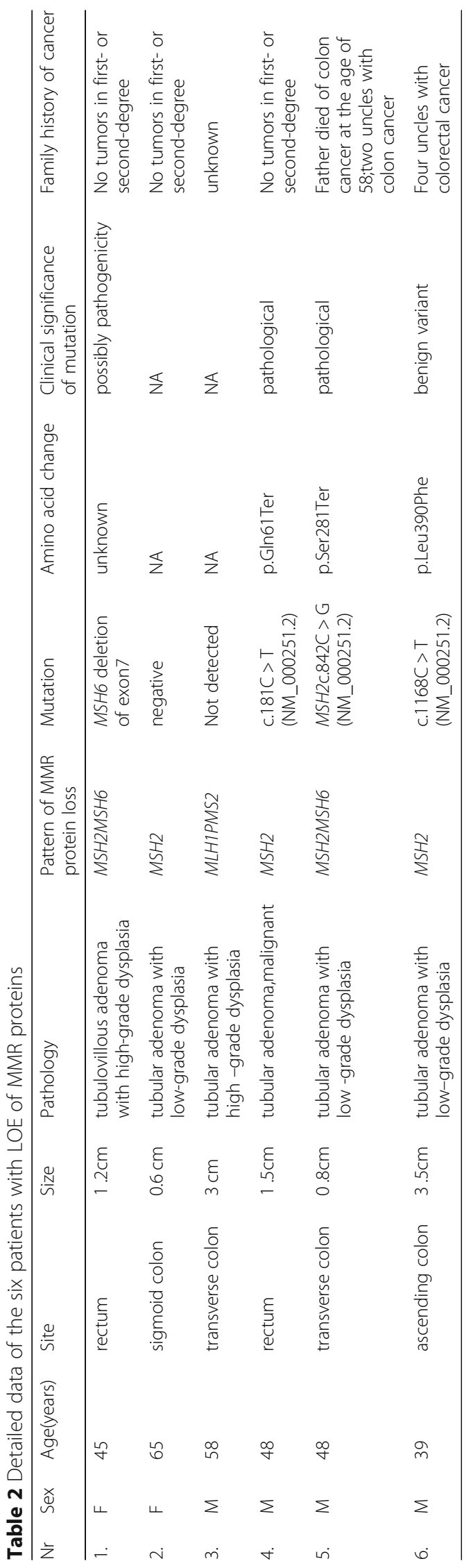




\section{Identification of LS}

In 6 patients with LOE of MMR proteins, one patient refused to be tested for gene, and the other 5 patients were tested. Table 2 summarizes the results of genetic testing.

Three patients who underwent Sanger sequencing had three mutations in $M S H 2$, two pathogenic mutations (c.181C > T,c.842 C>G), and one benign mutation. A patient who received Sanger bidirectional sequencing showed a deletion of exon 7 in MSH6, but he declined further multiplex dependent probe amplification testing. So we speculated that he had a pathogenic mutation. Another patient received NGS did not have mutation in $\mathrm{MSH} 2$.

\section{Discussion}

In the past, the initial diagnosis of Lynch syndrome was based on family history of cancer, according to the Amsterdam II standard or the Bethesda revision criteria. The final diagnosis was established by identification of a germline heterozygous pathogenic mutation in $M L H 1$, $M S H 2$, or MSH6, or an EPCAM deletion upon molecular genetic testing. However, modern Chinese families tend to be small, which may lead to incomplete penetrance of MMR mutations that make it difficult to recognize LS. Studies have shown that these criterias are less sensitive and/or specific [11, 12]. Currently, guidelines and studies strongly recommend LS screening for all newly diagnosed colorectal cancer patients using MMR,IHC and/or MSI tests [13-15]. Early diagnosis of LS patients is important; Heikki and Jarvinen et al. have shown that 15-year follow-up of colonoscopy in LS families effectively reduced mortality by $65 \%[16]$.

Adenoma is the primary pre-malignant lesion of Lynch syndrome. In one study concerning MMR gene germline mutation carriers, MSI was detected at $44 \%$ in low-grade epithelial neoplasia, and $100 \%$ in high-grade dysplasiaThe frequency of MSI in LS-related adenoma was 41$80 \%$ [17-22], and the risk of MSI was 3.6-fold higher than that of non-carriers. By the age of 60, the cumulative rate of adenoma in the mutation carrier was $70 \%$ [19]. MSI is an early molecular event in the LS-related adenoma. In one study concerning MMR gene germline mutation carriers, MSI was detected at $44 \%$ in low-grade epithelial neoplasia, and 100\% in high-grade dysplasia, suggesting that the MSI may not be the first, but it is possible to accelerate the progression of the adenoma to the invasive cancer [17]. To better understand colorectal carcinogenesis in LS, Aysel Ahadova et al. demonstrated that LS colorectal cancers can develop through three pathways. Some colorectal cancers in LS can in fact grow out from MMR-proficient adenomas after secondary inactivation of the MMR system; however, a larger portion of cancers appears to develop from mismatch repair deficient crypt focus (MMR-DCF), either through an adenomatous phase or as nonpolypous lesions with immediate invasive growth [23]. Hence, dMMR is a key characteristic of LS-related colorectal adenoma.

Because, adenomas with LOE of MMR proteins evolve to adenocarcinoma quickly, we need an early screening strategy to identify these adenomas. However, there is no clear screening strategy for LS in colorectal adenomas. A study of adenoma characteristics of MMR germline mutation carriers found that these adenomas are more common in the right colon, are generally $\geq 7$ $\mathrm{mm}$, and consist of high-grade intraepithelial neoplasia [22]. Detection of LS by only performing MMR IHC and/or MSI testing of adenomas in young patients may not be the best strategy. In patients with colorectal adenoma $<40$ years old, MMR IHC and MSI showed no abnormal results [7]. In 208 patients $<40$ years-old with colorectal adenoma, only one patient $(0.4 \%)$ exhibited LOE of MMR (MLH1 PSM2) proteins [10]. In our study, we found that the LOE of MMR proteins was mainly distributed in MSH-2 and MSH- 6 in 6 adenomas by MMR IHC detection in 508 inpatients with adenomas. There was no difference in the distribution of the left and right hemicolon among the six adenomas. The average age was 50 years, the mean size of adenoma was $1.7 \mathrm{~cm}$. Two adenomas showed high-grade dysplasia, and one adenoma showed malignant component. The remaining two adenomas showed no progression, but came from young patients with a family history of cancer and met the Bethesda revised criteria. Age of onset and the size of adenomas may be key factors in predicting LOE of MMR proteins, but a good strategy for detection of LS also needs to include advanced histology (high-grade dysplasia and malignant components) and a family history of colorectal cancer.

Ultimately, five patients underwent genetic testing. Two patients with LOE of the $M S H 2$ protein had pathogenic mutations,c. $181 \mathrm{C}>\mathrm{T}$ and c. $842 \mathrm{C}>\mathrm{G}$. Amino acid changes were p.Gln61Ter and p.Ser281Ter, which caused termination of amino acid translation. One case of MSH 6 exon 7 deletion mutation. The patient refused to further verify the multiple -dependent probe amplification test. Three cases were younger than 50 years old, 2 cases had advanced histology (high-grade dysplasia and malignant components), and 1 case had a family history of cancer.

Our study has limitations. First, MMR-deficient nonpolypous is another important precursor in LS; so our screening for LS from adenomatous polyps indeed may have missed quite a few cases. Second, our immunohistochemical screening strategy may also missed a few cases; genetic testing of all adenomatous polyps is the most accurate strategy. 


\section{Conclusion}

Currently, the study contains the largest sample size, screening for Lynch syndrome by MMR IHC on colorectal polyps. The results suggest that when young adenoma patients have a family history of LS associated cancer, or young adenoma patients have high risk characteristics, such as large volume, high grade dysplasia and malignant components, it may be reasonable to evaluate LS.

\section{Abbreviations}

dMMR: Deficient mismatch repair; IHC: Immunohistochemical; LOE: Loss of expression; LS: Lynch syndrome; MMR: Mismatch repair; MSI: Microsatellite instability; NGS: Next generation sequencing

\section{Acknowledgements}

Not applicable.

\section{Funding}

The cost of genetic testing for this study was supported by a Key project of Zhejiang medical and health science and technology platform plan (201481997)

\section{Availability of data and materials}

The datasets used and/or analyzed during the current study are available from the corresponding author upon reasonable request.

\section{Authors' contributions}

FCZ and JP participated in the design of the study and coordination, performed statistical analysis, interpreted and drafted the manuscript. DP and FCZ participated in data collection. QY performed MMR IHC, HZ was in charge of drawing blood, PSX performed the genetic testing. All authors read and approved the final manuscript

\section{Ethics approval and consent to participate}

This study was approved by the WenZhou Central Hospital ethics committee. All participants provided written informed consent.

\section{Consent for publication}

Not applicable.

\section{Competing interests}

The authors declare that they have no competing interests.

\section{Publisher's Note}

Springer Nature remains neutral with regard to jurisdictional claims in published maps and institutional affiliations.

\section{Author details}

'Department of gastroenterology, WenZhou Central Hospital, WenZhou, ZheJiang Province, China. ${ }^{2}$ Department of Pathology, WenZhou Central Hospital, WenZhou, ZheJiang Province, China. ${ }^{3}$ HangZhou Zhiyuan Medical Laboratory Co., Ltd, WenZhou, ZheJiang Province, China.

Received: 30 August 2018 Accepted: 4 March 2019

Published online: 12 March 2019

\section{References}

1. Mecklin JP. Frequency of hereditary colorectal carcinoma. Gastroenterology. 1987:93:1021-5

2. Jass JR, Stewart SM, Stewart J, Lane MR. Hereditary non-polyposis colorectal cancer--morphologies, genes and mutations. Mutat Res. 1994;310(1):125-33.

3. Pino MS, Mino-Kenudson M, Wildemore BM, Ganguly A, Batten J, Sperduti I, et al. Deficient DNA mismatch repair is common in lynch syndromeassociated colorectal adenomas. J Mol Diagn. 2009:11(3):238-47.

4. Yurgelun MB, Goel A, Hornick JL, Sen A, Turgeon DK, et al. Microsatellite instability and DNA mismatch repair protein deficiency in lynch syndrome colorectal polyps. Cancer Prev Res (Phila). 2012;5(4):574-82.
5. Walsh MD, Buchanan DD, Pearson SA, Clendenning M, Jenkins MA, Win AK, et al. Immunohistochemical testing of conventional adenomas for loss of expression of mismatch repair proteins in lynch syndrome mutation carriers: a case series from the Australasian site of the colon cancer family registry. Mod Pathol. 2012;25(5):722-30.

6. Loukola A, Salovaara R, Kristo P, Moisio AL, Kääriäinen $H$, Ahtola $H$, et al. Microsatellite instability in adenomas as a marker for hereditary nonpolyposis colorectal cancer. Am J Pathol. 1999;155(6):1849-53.

7. Velayos FS, Allen BA, Conrad PG, Gum J Jr, Kakar S, Chung DC, et al. Low rate of microsatellite instability in young patients with adenomas: reassessing the Bethesda guidelines. Am J Gastroenterol. 2005:100(5):1143-9.

8. Koh DC, Luchtefeld MA, Kim DG, Attal H, Monroe T, Ingersoll K. Microsatellite instability and MLH1 hypermethylation - incidence and significance in colorectal polyps in young patients. Color Dis. 2007;9(6):521-6.

9. Dow E, Buchanan DD, Winship IM. The utility of immunohistochemistry for mismatch repair proteins on colorectal polyps in the familial cancer clinic. Intern Med J. 2018. https://doi.org/10.1111/imj.13953.

10. Mendelsohn RB, Herzog K, Shia J, Rahaman N, Stadler ZK, Shike M. Molecular screening for lynch syndrome in young patients with colorectal adenomas. Clin Colorectal Cancer. 2017;16(3):173-7. https://doi.org/10.1016/ j.clcc.2017.01.002.

11. Hampel H, Frankel WL, Martin E, Arnold M, Khanduja K, Kuebler P, et al. Screening for the lynch syndrome (hereditary nonpolyposis colorectal cancer). N Engl J Med. 2005;352(18):1851-60.

12. Barnetson RA, Tenesa A, Farrington SM, Nicholl ID, Cetnarskyj R, Porteous $M E$, et al. Identification and survival of carriers of mutations in DNA mismatch-repair genes in colon cancer. N Engl J Med. 2006;354(26):2751-63.

13. Evaluation of Genomic Applications in P, Prevention Working G. Recommendations from the EGAPP working group: genetic testing strategies in newly diagnosed individuals with colorectal cancer aimed at reducing morbidity and mortality from lynch syndrome in relatives. Genet Med. 2009;11(1):35-41.

14. Giardiello FM, Allen Jl, Axilbund JE, Boland CR, Burke CA, Burt RW, et al. Guidelines on genetic evaluation and management of lynch syndrome:a consensus statement by the US multi-society task force on colorectal cancer. Gastroenterology. 2014;147(2):502-26.

15. Rubenstein JH, Enns R, Heidelbaugh J, Barkun A, Clinical Guidelines C. American Gastroenterological Association Institute guideline on the diagnosis and management of lynch syndrome. Gastroenterology. 2015; 149(3):777-82.

16. Jarvinen $\mathrm{HJ}$, Markkuaarnio $\mathrm{H}$, et al. Controlled 15-year trial on screening for colorectal cancer in families with hereditary nonpolyposis colorectal cancer. Gastroenterology. 2000;118:829-34.

17. Rijcken $\mathrm{FE}, \mathrm{Hollema} \mathrm{H}$, Kleibeuker $\mathrm{JH}$, et al. Proximal adenomas in hereditary non-polyposis colorectal cancer are prone to rapid malignant transformation. Gut. 2002:50:382-6.

18. Akiyama $Y$, Iwanaga $R$, Saitoh $K$, et al. Transforming growth factor beta type II receptor gene mutations in adenomas from hereditary nonpolyposis colorectal cancer. Gastroenterology. 1997;112:33-9.

19. De jong $A E$, Morreau $H$, Van Puijenbroek $M$, et al. The role of mismatch repair gene defects in the development of adenomas in patients with HNPCC. Gastroenterology. 2004;126:42-8.

20. Aaltomen LA, Peltomaki P, Mecklin JP, et al. Replication errors in benign and malignant tumors from hereditary nonpolyposis colorectal cancer patients. Cancer Res. 1994:54:1645-8.

21. lino $H$, Simms L, Young J, et al. DNA microsatellite instability and mismatch repair protein loss in adenomas presenting in hereditary non-polyposis colorectal cancer. Gut. 2000;47:37-42

22. Kalady MF, Kravochuck SE, Heald B, et al. Defining the adenoma burden in lynch syndrome. Dis Colon Rectum. 2015;58(4):388-92.

23. Ahadova A, Gallon R, Gebert J, et al. Three molecular pathways model colorectal carcinogenesis in lynch syndrome. Int JCancer. 2018;143:139-50. 